\title{
Imaging and spectroscopic studies of Haro 6-19 (HH319) ${ }^{\star}$
}

\author{
T. Yu. Magakian ${ }^{1}$, T. A. Movsessian ${ }^{1}$, K. Ogura ${ }^{2}$, and V. L. Afanasiev ${ }^{3}$ \\ 1 Byurakan Astrophysical Observatory, Aragatsotn prov., 378433 Armenia and Isaac Newton Institute of Chile, \\ Armenian Branch \\ e-mail: tigmov@bao.sci.am \\ 2 Kokugakuin University, Higashi, Shibuya-ku, Tokyo 150-8440, Japan \\ e-mail: ogura@kokugakuin.ac.jp \\ 3 Special Astrophysical Observatory, N.Arkhyz, Karachaevo-Cherkesia 369167, Russia \\ e-mail: vafan@sao.ru
}

Received 31 August 2001 / Accepted 24 October 2001

\begin{abstract}
We performed morphological and spectro-imaging investigations of the little-studied HH object Haro 6-19 (HH319). Besides the main object, two other small HH knots were found. The images of the object in various spectral lines and maps of radial velocity and electronic density are presented. Either of the T Tauri stars FY and FZ Tau is suggested as the possible exciting source. The spatial distribution of the density and radial velocity in the object, combined with other evidence, shows that HH319 could be a shocked cloudlet. In many aspects HH319 is similar to HH29 in L1551.
\end{abstract}

Key words. ISM: individual objects: HH319 - Herbig-Haro objects - ISM: kinematics and dynamics

\section{Introduction}

Located in the eastern part of the Heiles Cloud 2 of the Taurus Molecular Cloud (TMC), Haro 6-19 is a HerbigHaro (HH) object little studied up to now. It is included in the catalogue of $\mathrm{HH}$ objects compiled by Reipurth (1999) as HH319 according to a private communication from Mundt, but no detailed spectral data or even images have been published so far. Its source of excitation is also unknown.

Haro 6-19 was discovered by Haro et al. (1953) in a search for $\mathrm{H} \alpha$ emission objects in Taurus. Along with other small nebulae in the Taurus dark cloud, it was included in the list of cometary nebulae of Badalian (1960) as Bad 2324. It should be noted that Haro 6-19 was also rediscovered as a fast-moving nebulous object by Luyten (1971) as LP 358-405. However, in both the articles its identity with Haro 6-19 was unnoticed. After these, no references to this object could be found in published papers.

We considered this object as a sufficiently bright and interesting target to study in detail in order to better

\footnotetext{
Send offprint requests to: T. Yu. Magakian,

e-mail: tigmag@sci.am

* Partly based on observations collected with the $6 \mathrm{~m}$ telescope of the Special Astrophysical Observatory (SAO) of the Russian Academy of Sciences (RAS) which is operated under the financial support of Science Department of Russia (registration number 01-43).
}

understand its properties. We carried out narrow-band imaging as well as 3D spectroscopy with a multi-pupil spectrograph.

\section{Observations}

Direct imaging of the HH319 field was performed on 12 October and 20 November 1998 on the $2.6 \mathrm{~m}$ telescope of Byurakan Astrophysical Observatory with the ByuFOSC-2 spectral camera, equipped with a $1024 \times$ 1024 CCD (Movsessian et al. 2000). We obtained images behind $\mathrm{H} \alpha$ and $[\mathrm{SII}]$ narrow-band filters as well as in the $I$ band.

Integral-field spectral observations were performed on 26 November 1998 on the $6 \mathrm{~m}$ telescope of the Special Astrophysical Observatory (Russia) with a new multi-pupil fiber spectrograph (MPFS) and a $1034 \times$ 1034 CCD as light-receiver. The spectral resolution was about $1.3 \AA$ /pix. On the input matrix of $15 \times 16$ pupils (each was equal to 1 arcsec on the sky) we placed the brightest (south-eastern) part of the object.

For the direct images the cosmic ray removal and flat-fielding was done with the aid of the MIDAS system. For the 3D-spectra we used dedicated software packages, developed by one of authors (VLA) in the IDL environment; the operations included specific data extraction from the 3D-spectra, wavelength calibration of individual vectors, correction of fiber transparency variations, and 


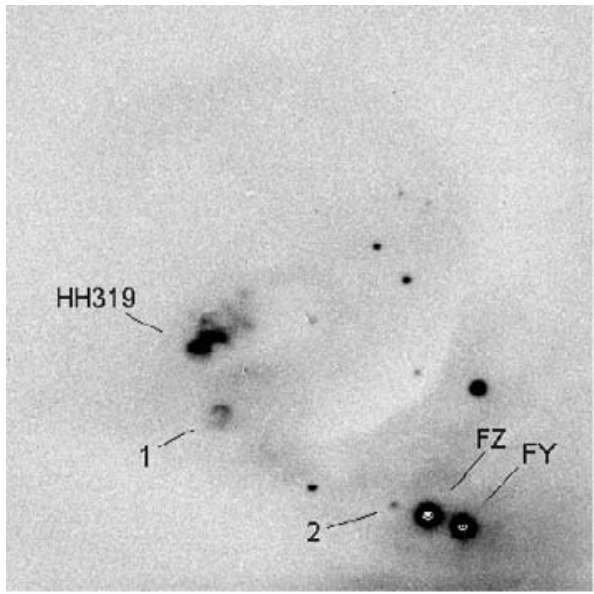

a)

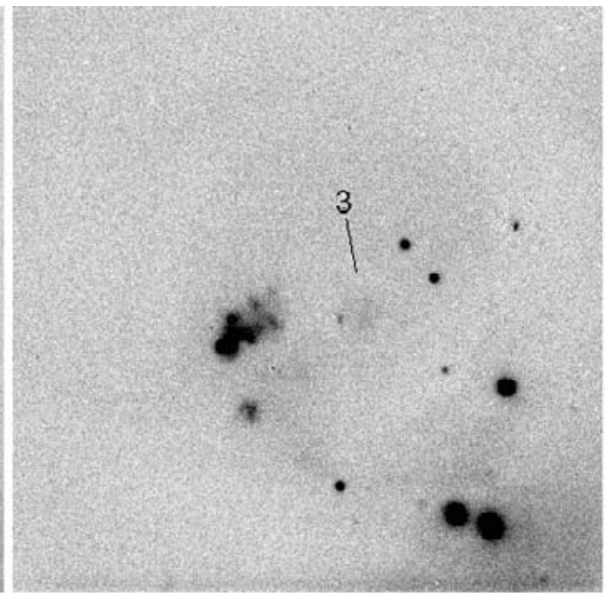

b)

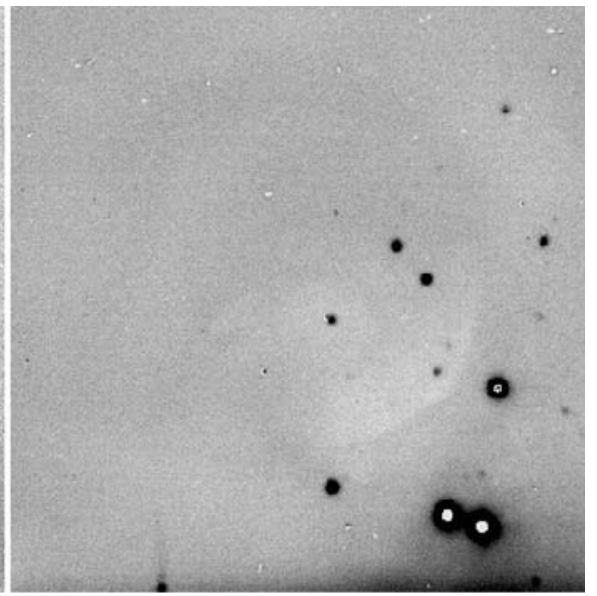

c)

Fig. 1. Direct images of the HH319 field in $\mathrm{H} \alpha$ a), $\left[\mathrm{S}_{\mathrm{II}}\right] 6717 / 6731$ b) and the $I$ band $\left.\mathbf{c}\right)$. Objects mentioned in the text are marked. The field shown is approximately $4^{\prime} \times 4^{\prime}$.

finally, mapping of the objects in various spectral lines and parameters.

\section{Results}

\subsection{Direct imaging}

On the direct images (Fig. 1) the HH nature of HH319 is obvious. The object is totally invisible in the image obtained with the $I$ filter. There are no faint stars embedded in the immediate vicinity, either. In fact, the field is totally obscured. On the $\mathrm{H} \alpha$ and $[\mathrm{S} \mathrm{II}]$ images HH319 is prominent. As a whole it has an elongated, somewhat arrow-like shape and consists of several knots, the brightest of which is located in the SE part of the object. To the south of the main object another separate HH knot (marked as object "1" in Fig. 1a) is visible. In $\mathrm{H} \alpha$ it is much brighter than in [S II], and has a bubble-like appearance, the northern rim being the brightest.

These knots are surrounded by faint nebular wisps of a reflecting nature, mixed with several very dark globules, well seen in the $I$ image. Two pairs of nearby bright stars are illuminating the separate reflection nebulae DG37 and DG38. The stars in DG38 are known as the variables FY Tau and FZ Tau, belonging to the T Tau class.

There is one more very small but bright, especially in $\mathrm{H} \alpha$, emission knot near FZ Tau (marked as object "2" in Fig. 1a), which probably is yet another $\mathrm{HH}$ object, but its connection with HH319 is not clear. It could also represent an outflow from FZ Tau.

Besides, one can note very faint emission patches that are traceable from FY/FZ Tau in the direction of HH319. As a whole they outline an elliptically elongated loop (with dimensions about $2^{\prime} \times 1^{\prime}$ ) with HH319 near one end. Their nature is not clear. One of them, located to the west of HH319, is definitely brighter in the [SII] image (marked as object "3"; compare Figs. 1a and b). The others are brighter in $\mathrm{H} \alpha$.

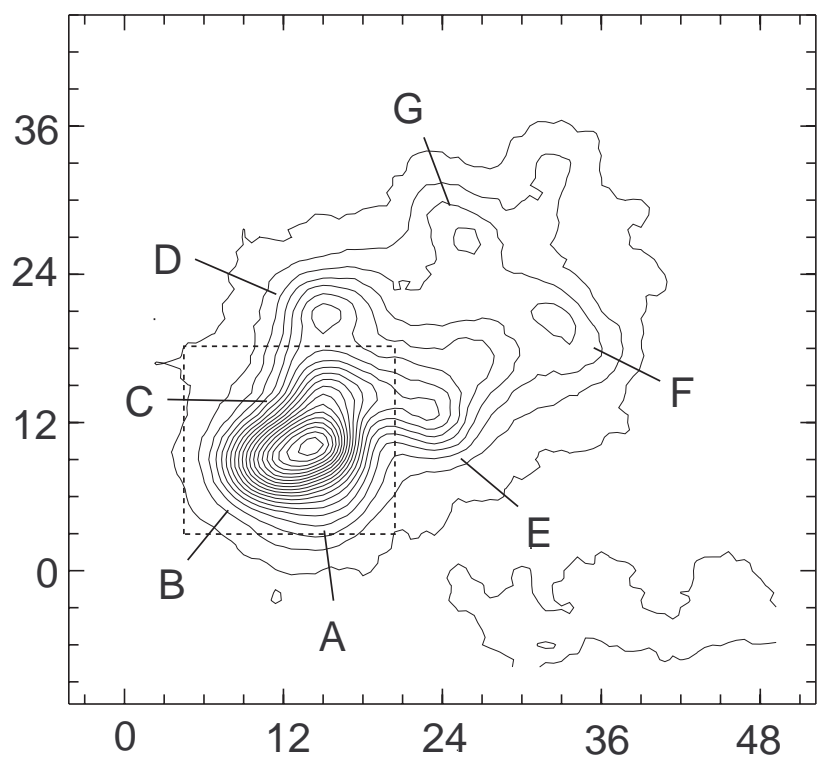

Fig. 2. Isocontours of the direct image of HH319 in [S II]6717/6731, showing its inner structure. Main detailes are labelled. The units in both axes is arcseconds. The area observed with MPFS is denoted by the rectangle.

Of course, morphological analysis alone is not sufficient to identify one of these stars as the probable excitation source of HH319, but it is worth mentioning that the position angle of the proper motion of $\mathrm{HH} 319\left(72^{\circ}\right.$, according to Luyten 1971) is oriented away from FY/FZ Tau.

When comparing the images in $\mathrm{H} \alpha$ and $[\mathrm{S} \mathrm{II}]$ one can easily note the differences in excitation of the various details. Besides, in the $[\mathrm{S} \mathrm{II}]$ image the whole body of HH319 is more crisply shaped and its knotty structure is more prominent than in $\mathrm{H} \alpha$. At least 6-7 well defined knots can be identified (Fig. 2). We marked these knots with the letters A-G.

Coordinates of all the above-mentioned objects were measured on the DSS-2 image with the aid of the 
Table 1. Coordinates of the objects, 2000.0.

\begin{tabular}{lll}
\hline Object & RA & Dec \\
\hline FY Tau & 043230.7 & 241956 \\
FZ Tau & 043231.9 & 242002 \\
Obj.1 & 043238.8 & 242051 \\
Obj.2 & 043232.9 & 242006 \\
Obj.3 & 043234.9 & 242136 \\
HH319A & 043239.4 & 242124 \\
HH319B & 043239.7 & 242123 \\
HH319C & 043239.4 & 242127 \\
HH319D & 043239.3 & 242135 \\
HH319E & 043239.0 & 242127 \\
HH319F & 043238.2 & 242133 \\
HH319G & 043238.7 & 242143 \\
\hline
\end{tabular}

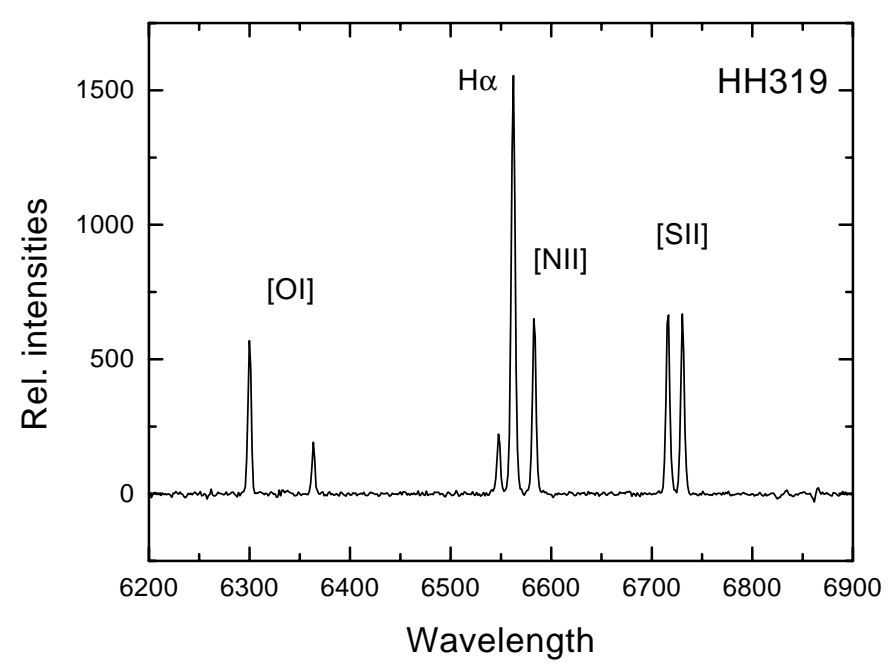

Fig. 3. Integral spectrum of HH319 in relative intensities.

FITSVIEW program. They are given in Table 1. We estimate that the positional errors of these measurements are about $1.5^{\prime \prime}$; of course, for the faint nebulous knots they could be higher.

\subsection{Integral-field spectroscopy}

As a whole, HH319 has a normal spectrum of a moderateexcitation $\mathrm{HH}$ object, with prominent emission lines of $\mathrm{H} \alpha$, [S II]6717/6731, [N II]6548/6584, [O I]6300/6363 and traces of a very faint continuum. The integral spectrum of the main body of HH319, obtained by summation of 42 individual multi-pupil spectra, is shown in Fig. 3.

On the other hand, certain variations in relative intensity and velocity of emission lines exist among individual spectra. To better represent this, we display in Fig. 4 the maps of the observed part of the object in all the main lines: $\mathrm{H} \alpha$, [S II]6717 and 6731, [N II]6583 and [O I]6300. Note that they includes knots A, B and C.

As can be seen from Fig. 4, these maps are in remarkable agreement with direct images. Especially the more or less smooth appearance of the object in $\mathrm{H} \alpha$, compared with the knotty structure in [SII] and [N II] should be noted. Another interesting feature of these maps is that the brightest part of the object in $\mathrm{H} \alpha$ and [O I] is knot $\mathrm{A}$, but in $[\mathrm{S} \mathrm{II}]$ and $[\mathrm{NII}]$ knot B has nearly the same brightness as knot $\mathrm{A}$. Thus, the ratio $[\mathrm{S} \mathrm{II}] / \mathrm{H} \alpha$ in this knot must be higher, and, consequently, its excitation be lower, than in knot A. Knot C, though fainter than A and B, also is better seen in [S II] and [N II].

Virtually everywhere in the studied area all emission lines are single-peaked and narrow, with FWHM not exceeding the instrumental profile. However, definite variations exist in other physical parameters. This can be seen in Fig. 5, where the maps of electronic density, estimated from the ratio of the $[\mathrm{S} \mathrm{II}] \lambda \lambda 6717 / 6731$, and of heliocentric radial velocity, measured by the same lines are shown. As we see, the radial velocity of the object is low (from -10 to $-20 \mathrm{~km} \mathrm{~s}^{-1}$ nearly everywhere). Its highest values (up to $-50 \mathrm{~km} \mathrm{~s}^{-1}$ ) are observed near knot A (actually between knot A and the edge of the object). The same could be said about the distribution of electron density: it also peaks near knot A, with a shift to the object's edge.

\section{Discussion and conclusion}

As was already mentioned before, the results of the direct imaging and the field spectroscopy of HH319 are in good agreement. The whole set of data shows that HH319 is a very typical $\mathrm{HH}$ object in its physical parameters. Combining the morphological and proper motion data, it is possible to draw the conclusion that the source of excitation of HH319 could be either FZ or FY Tau. In this case we can consider the very faint, elliptical emission loop between HH319 and these stars as the walls of a bubble, produced by the outflow.

We can then identify knot $A$ with the apex of the shock, because it is the brightest part of HH319, has the highest excitation and, nearly coincides with the peaks of the density and velocity. However, the most intriguing feature is that these peaks are located on the "trailing" side of the $\mathrm{HH}$ object as a whole and, particularily, of knot A, i.e. opposite to the proper motion vector. But just this side is the nearest to $\mathrm{FY} / \mathrm{FZ}$ Tau and, besides, has the steepest intensity gradient (see Fig. 2). Actually, all three knots $\mathrm{A}, \mathrm{B}$ and $\mathrm{C}$ collectively resemble a bow shock, oriented towards the FY/FZ Tau.

Such a picture is not too common and could be understood if this $\mathrm{HH}$ object represents a so-called "shocked cloudlet" (Schwartz 1978).

Another piece of evidence for this explanation could be the low velocity of HH319 as a whole. The proper motion of $0.05^{\prime \prime} /$ year given by Luyten (1971) corresponds to a tangential velocity of $30 \mathrm{~km} \mathrm{~s}^{-1}$ at the distance of $140 \mathrm{pc}$ of TMC, which is of the same order as our radial velocity estimates. Taking, for example, $40 \mathrm{~km} \mathrm{~s}^{-1}$ as the mean radial velocity, we will obtain $50 \mathrm{~km} \mathrm{~s}^{-1}$ as the upper limit for the full space velocity and $53^{\circ}$ for the angle of the flow with respect to the plane of the sky. Of course, 


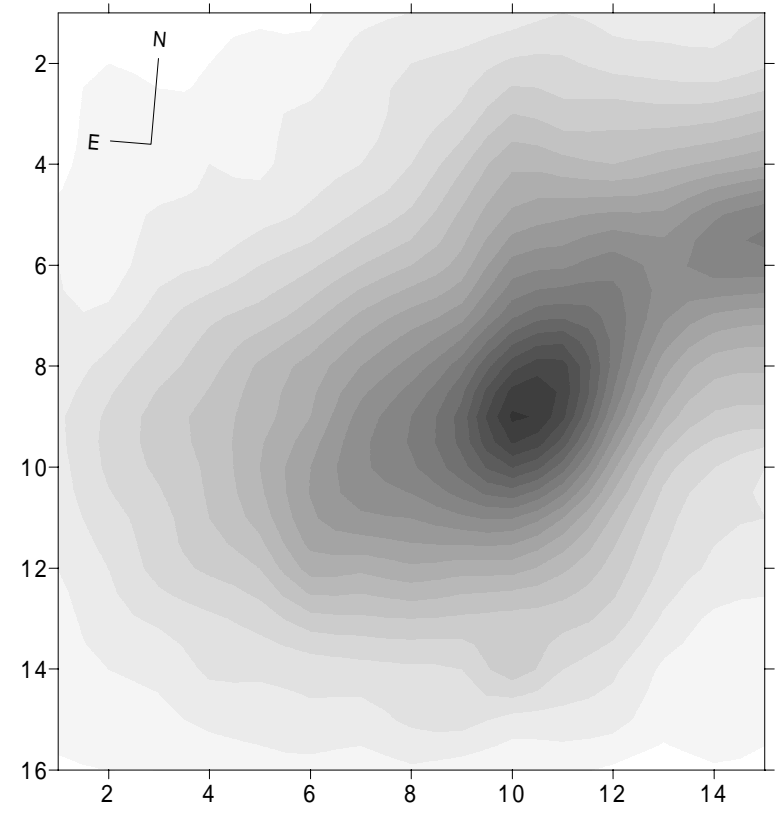

a

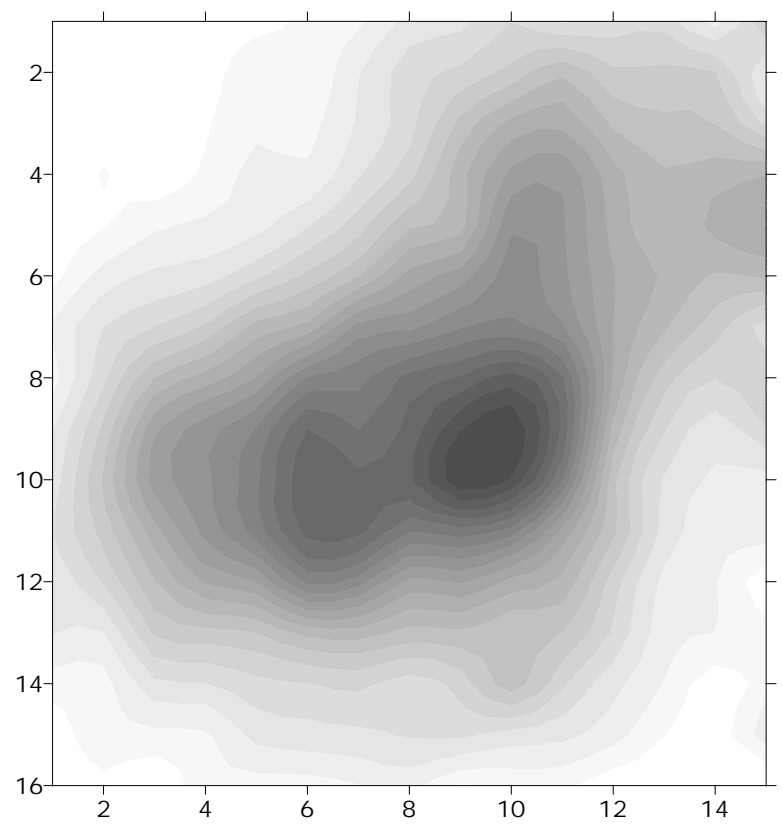

C
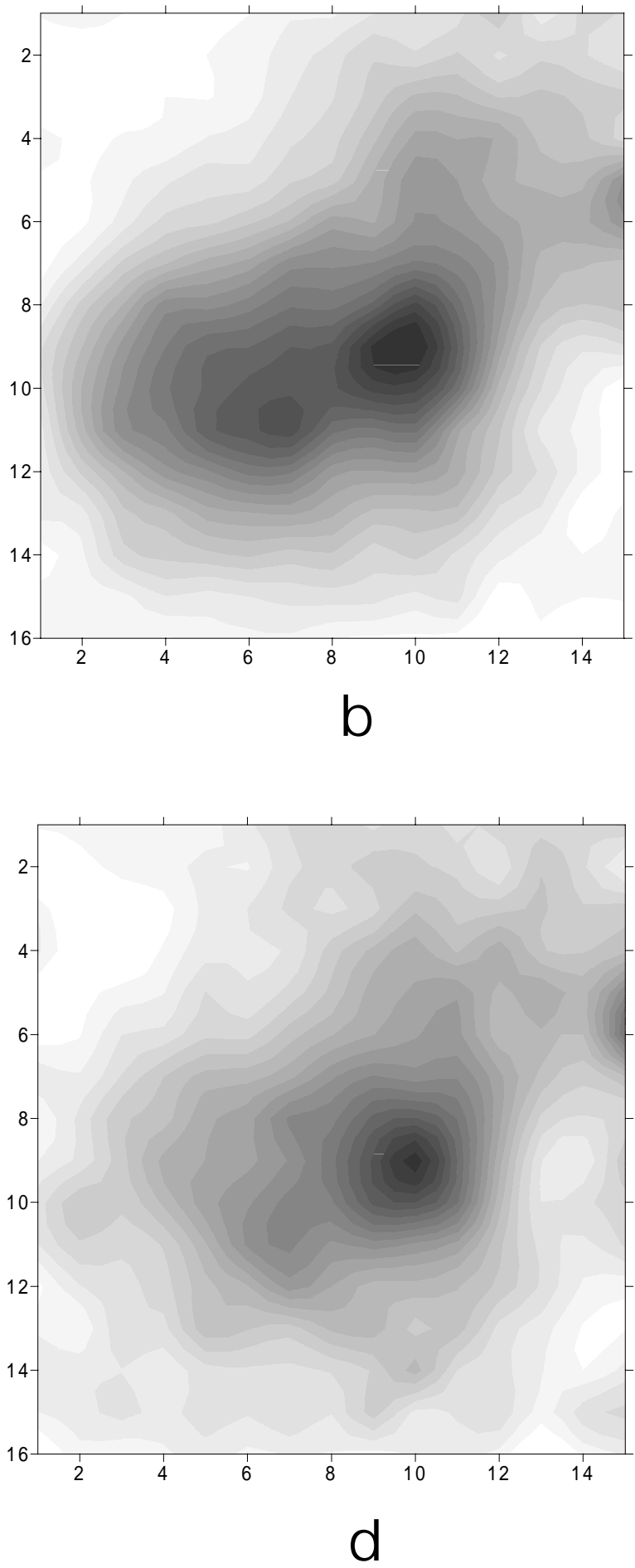

Fig. 4. Maps of the brightest part of HH319 in $\mathrm{H} \alpha \mathbf{a}$ ), [N II]6583 b), [S II]6717/6731 c) and [O I]6300 d) emission lines, constructed from the 3D spectra. Gradation steps for each image are $5 \%$ of the total range of intensities.

$50 \mathrm{~km} \mathrm{~s}^{-1}$ is a rather low value to explain the excitation level of HH319, but this can be understood if this object is indeed the shocked cloudlet.

Comparing HH319 with other HH objects, we found that it has a rather strong resemblance with HH29 in L1551, which, despite its higher excitation and velocity, has a very similar morphology and distribution of physical parameters. Just these features allowed investigators to interpret HH29 as a "shocked cloudlet" (Fridlund et al. 1998).

Of course, in order to confirm this conclusion, new, more precise proper motion measurements are needed. It will be interesting also to obtain the physical parameters of other knots. As a candidate "shocked cloudlet", which appears to be a rare type of HH object, HH319 is an interesting target for further studies. 

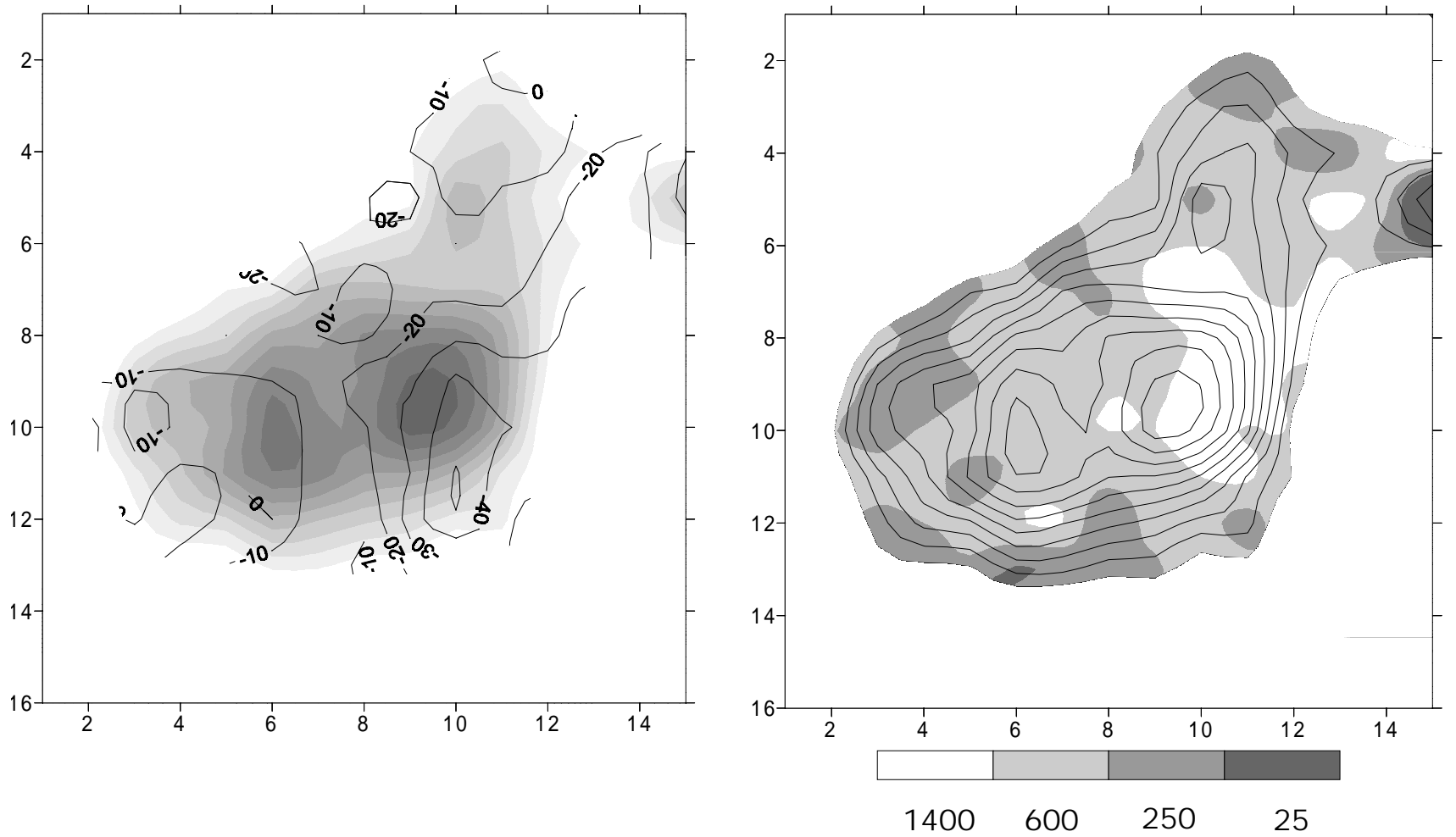

Fig. 5. Maps of the distributions of radial velocity (left) and electron density (right) estimated from the [S II] lines. Both are overlayed on the [S II] image of HH319, shown by gray scale in the left map and by contours in the right map.

Acknowledgements. We thank the referee Dr. J. Eislöffel for the helpful comments and suggestions.

\section{References}

Badalian, G. S. 1960, Dokl. AN Arm. SSR, 31, 261

Fridlund, C. V. M., Liseau, R., \& Gullbring, E. 1998 A\&A, 330,327
Haro, G., Iriarte, B., \& Chavira, E. 1953, Bol. Obs. Tonantzintla y Tacubaya, 8,3

Luyten, W. J. 1971, The Hyades (Univ. of Minnesota, Minneapolis), 81

Movsessian, T. A., Boulesteix, J., Gach, J.-L., et al. 2000, Baltic Astron., 9, 652

Reipurth, B. 1999, A General Catalogue of Herbig-Haro Objects, 2nd edition (electronic version)

Schwartz, R. D. 1978, ApJ, 223, 884 